\title{
Programa de orientação desenvolvido com adolescentes em centro de saúde: conhecimentos adquiridos sobre os temas abordados por uma equipe multidisciplinar
}

\author{
Program developed with adolescents \\ in a health center: acquired knowledge about \\ the themes studied by a multidisciplinary staff
}

Dulce Méri Tótola Faustini 1

Neil Ferreira Novo 2

Maria Cristina Faria da Silva Cury 3

Yara Juliano 2

\footnotetext{
1 Centro de Saúde

da Universidade de Santo Amaro. Rua Professor Enéas de Siqueira Neto, 340, Jardim das Imbuias, 04829-300, São Paulo SP. jsoliveira@unisa.br

2 Departamento de Saúde Coletiva da Faculdade de Medicina da Universidade de Santo Amaro/Unisa.

3 Coordenação do curso de Pós-graduação em Saúde Materno-Infantil da Universidade de Santo Amaro.
}

\begin{abstract}
Fifty-nine adolescent were analyzed in this study consisted of 37 girls and 22 boys who looked for help at the school-health center at Santo Amaro University. This study aimed verifying in the orientation program developed with the adolescents promoted knowledge about the questions developed during the execution of the referred program (sexuality, sexually transmitted diseases-STD, AIDS, drugs). The adolescents completed an evaluation questionnaire, which included the questions that subsequently would be asked before beginning the program. At the end of the process, they completed the evaluation questionnaire again. The comparison between the previous and post-program answers served as an assessment of the knowledge acquired by the adolescents. The results showed a significant increase of knowledge about the themes developed by a multidisciplinary staff and the gender did not differ significanthy in relation to the acquired knowledge.
\end{abstract}

Key words Adolescence, Multidisciplinary service, Adolescence-health
Resumo Foram estudados 59 adolescentes, sendo 37 do sexo feminino e 22 do sexo masculino, que procuraram atendimento no Centro de Saúde Escola da Universidade de Santo Amaro. O objetivo foi verificar se o programa de orientação desenvolvido com adolescentes promoveu conhecimento sobre as questões abordadas durante a realização do referido programa (sexualidade, doenças sexualmente transmissíveis, Aids, drogas). Antes da realização do programa, os adolescentes responderam a um questionário avaliativo contendo questões com temas específicos dessa fase da vida. No final do processo, o questionário foi novamente respondido. O confronto entre as respostas pré e pós-programa serviu como aferição do conhecimento adquirido pelos adolescentes. Os resultados mostraram aumento significativo de conhecimento após o programa desenvolvido por uma equipe multidisciplinar e que os sexos não diferiram significantemente em relação aos conhecimentos adquiridos. Palavras-chave Adolescência, Atendimento multidisciplinar, Adolescência-saúde 


\section{Introdução}

O conceito da adolescência é bastante recente, estando relacionado ao processo de industrialização, urbanização e modernização, próprio das sociedades ocidentais contemporâneas. $\mathrm{O}$ universal interesse pelo estudo da adolescência na atualidade advém tanto da explosão demográfica do pós-guerra, que teve como conseqüência imediata significativo crescimento percentual da população jovem no mundo, como a ampliação da faixa etária com as características da adolescência (Osório, 1989).

Do ponto de vista cronológico, a Organização Mundial da Saúde define a adolescência como sendo a faixa etária de 10 a 20 anos (Coli, 1994). De acordo com o critério físico, a adolescência abrange as modificações anatômicas e fisiológicas que transformam a criança em adulto, correspondendo ao período que vai desde o aparecimento dos caracteres sexuais secundários e início da aceleração do crescimento, até o apogeu do desenvolvimento com a parada do crescimento (Coli, 1994).

Segundo o ponto de vista psicológico, o adolescente passa por desequilíbrios e instabilidades extremas. Em nosso meio cultural, mostra períodos de elação, de introversão, alternando com audácia, timidez, descoordenação, urgência, desinteresse ou apatia, que se sucedem ou são concomitantes com conflitos afetivos, crises religiosas, nas quais se pode oscilar do ateísmo anárquico ao misticismo fervoroso, intelectualizações e postulações filosóficas, ascetismo, condutas sexuais dirigidas para o heteroerotismo e até a homossexualidade ocasional. O que configura uma entidade semipatológica, que denominam "síndrome normal da adolescência", que é perturbada e perturbadora para o mundo adulto, mas necessária, absolutamente necessária, para o adolescente, que neste processo vai estabelecer a sua identidade, sendo este o objetivo fundamental deste momento da vida (Aberastury e Knobel, 1992). Para esses mesmos autores, o adolescente realiza três lutos fundamentais: a) o luto pelo corpo infantil perdido; b) o luto pelo papel e identidade infantis; c) o luto pelos pais na infância.

Por fim, do ponto de vista social, a adolescência corresponde a um período da vida, no qual o indivíduo perde direitos e privilégios de criança e começa a assumir direitos e responsabilidades de adulto (Coli, 1994).

Para Donas (1992), na adolescência o processo de desenvolvimento adquire característi- cas muito especiais: a busca de identidade, de independência criatividade, auto-estima, juízo crítico, sensibilidade, afetividade, elaboração de um projeto de vida, sexualidade e educação. O produto final do acontecer de todas as características leva o adolescente a definir o que considera um fato fundamental: o seu estilo de vida. Os jovens que desenvolvem um estilo de vida de alto risco, segundo Donas, são aqueles que têm maiores probabilidades de padecer de problemas que julgamos os grandes problemas da adolescência, ou seja, os distúrbios de afetividade e de conduta, os distúrbios da aprendizagem, a gravidez indesejada, os problemas da violência social e ecológica, as doenças sexualmente transmissíveis, os acidentes que conduzem a seqüelas e mortes, suicídio drogas, etc. Enquanto os de baixo risco são aqueles que não têm as características e as atividade que os expõem a desenvolver tais doenças, estados patológicos ou grandes distúrbios no processo da adolescência.

Sousa (1988) afirmou que a educação sexual deve começar no lar, antes mesmo da criança ingressar na escola. Deve ser continuada durante o desenvolvimento, e precisa ser aprimorada na adolescência.

Pilon (1986) observou que os jovens possuem informações incompletas a respeito de reprodução humana e da sexualidade, fato que se agrava quando se refere ao sexo oposto (em que é mais falha).

Em relação à anticoncepção, a Pesquisa sobre Saúde Reprodutiva e Sexualidade do Jovem (PSRSJ, 1989-1990) mostrou que a totalidade dos jovens conhece ou já ouviu falar sobre a pílula e o condom (acima de 97\%). A gestação na adolescência decorre, principalmente, da nãoutilização de qualquer método contraceptivo e, em menor porcentagem, da utilização inadequada desses métodos (Messina, 1993)

Pesquisas apontam que a atividade sexual tem se iniciado cada vez mais cedo. A desinformação (referente a ignorância da fisiologia da reprodução e das conseqüências das relações sexuais por parte de muitas adolescentes) e a falta de garantias psicológicas e sociais têm exposto o adolescente a riscos de saúde, envolvendo a anticoncepção e a concepção. A gravidez precoce é uma preocupação em vários países, e também no Brasil (Adolescência e Saúde, 1994).

Como conseqüência da gravidez na adolescência, a literatura registra maior mortalidade e morbidade maternas, baixo peso ao nascer e 
prematuridade e riscos mais elevados de morbidade e mortalidade infantil (Adolescência e Saúde, 1994). Tais problemas podem ser minimizados quando controlados por uma atenção pré-natal adequada e pelo acompanhamento dos filhos das mães adolescentes.

Segundo a Organização Mundial de Saúde (OMS) e Organização Pan-Americana de Saúde (Opas), a adolescência constitui um processo fundamental biológico e de vivências orgânicas, em que se acelera o desenvolvimento cognitivo e de estruturação da personalidade. A gravidez leva sérios riscos às adolescentes devido a sua imaturidade física e psicológica, falta de apoio dos familiares, início tardio do pré-natal ou mesmo a falta deste e as dificuldades em concluir seus estudos, o que afeta sua qualidade de vida, sua oportunidade de emprego e sua estabilidade financeira (Braden, 2000).

As estatísticas apontam, que a incidência das doenças sexualmente transmissíveis (DSTs) está em ascensão. Para Ribeiro (1998), a família tem papel fundamental na educação dos filhos para prevenção das doenças sexualmente transmissíveis. A falta de conhecimento sobre os sintomas por parte dos adolescentes, os tabus e a vergonha de procurar os serviços especializados retardam o tratamento, o que traz conseqüências de longo prazo como a esterilidade, abortamentos, problemas neurológicos e de infecções generalizadas (Adolescência e Saúde, 1994).

Chicrala et al. (1977) em um trabalho avançado no morro do Pau da Bandeira (bairro de Vila Isabel, Rio de Janeiro), onde foram entrevistados 142 adolescentes do sexo feminino $(84,0 \%)$ e 27 do sexo masculino (16,0\%), constataram que, dos 169 adolescentes estudados, 157 (92,9\%) já tinham ouvido falar de DSTs. A grande maioria dos adolescentes $(78,7 \%)$ reconheceu pelo menos uma via de transmissão das DSTs, sendo citadas: via sexual $(97,7 \%)$, via sangüínea $(17,3 \%)$ e uso de drogas $(12,8 \%)$.

A grande maioria dos adolescentes brasileiros sobrevivem miseravelmente e marginalizados, excluídos das estatísticas da educação e do mercado de trabalho. Muitos trabalham de forma ilegal na América Latina, sem benefícios da previdência social, baixos salários e más condições de trabalho, o que contribui para um alto risco à saúde. As sucessivas crises econômicas nos países da região têm como conseqüência a diminuição do tempo para as tarefas escolares e o abandono precoce da escola para ir trabalhar (Benguigui et al., 1997).
A pobreza, a iniqüidade e a discriminação produzem e mantêm uma população em risco de padecer dos chamados "grandes problemas da adolescência”. O consumo de drogas é um dos principais problemas que a sociedade enfrenta atualmente e vem aumentando consideravelmente nas últimas décadas. Essa população está na mira do tráfico de drogas, pois é grande o espaço para o desenvolvimento dos que vivem dessa indústria e desse comércio. $\mathrm{O}$ uso excessivo de álcool aumentou na década de 1980. O consumo de tabaco, em contraste com a diminuição nos países desenvolvidos, está aumentando entre mulheres jovens. A droga ilícita mais usada é a maconha, que freqüentemente é consumida junto com o álcool e tabaco. Estima-se que de 10 a $30 \%$ dos adolescentes tenham tido a experiência de consumo. $\mathrm{O}$ uso de cocaína está aumentando na América Latina (Benguigui et al., 1997).

Scivoletto et al. (1996) estudaram 21 pacientes com idades entre 11 e 17 anos. O início do consumo de drogas ocorreu por volta de 11 anos com o uso de álcool, caminhando rapidamente para o tabaco, maconha, solventes, opióides, cocaína inalada e crack por volta dos 14 anos. A curiosidade em relação aos efeitos foi o principal fator alegado como motivador da experimentação de todas as substâncias e a pressão exercida pelo grupo de amigos foi importante para o início do consumo de álcool, maconha e solventes somente em cerca de $20 \%$ dos casos.

Os programas de educação para adolescentes são importantes na prevenção dos problemas específicos dessa fase da vida. Para que sejam implementados, é necessário conhecimento adequado desse grupo. O objetivo deste estudo foi avaliar o programa de orientação desenvolvido com adolescentes no Centro de Saúde Escola da Universidade de Santo Amaro sobre questões específicas dessa fase da vida como sexualidade, anticoncepção, gravidez, DSTs e drogas, com a finalidade de propiciar atitudes e práticas adequadas para o desenvolvimento de estilos de vida saudável.

\section{Material e método}

O trabalho foi realizado no Centro de Saúde Escola da Universidade de Santo Amaro (Unisa), no período de janeiro de 1997 a julho de 1999, com 59 adolescentes, sendo 37 do sexo feminino e 22 do sexo masculino. O critério de 
seleção foi o de adolescentes que procuraram atendimento na referida instituição.

Foram estudados cinco grupos constituídos por 12 adolescentes em média, com idade entre 12 a 19 anos. Cada encontro ocorreu uma vez por semana com duração de 90 minutos, e foi desenvolvido por uma equipe multiprofissional, que contou com a participação de um médico, um psicólogo, um assistente social, um nutricionista, um enfermeiro e um educador em saúde pública. Os membros da equipe participavam de todos os grupos.

A cada encontro foi abordado um tema. As discussões versaram sobre: crescimento e desenvolvimento, anatomia e fisiologia dos corpos feminino e masculino, sexualidade, gravidez, anticoncepção, doenças sexualmente transmissíveis e drogas. A apresentação dos temas era feita por meio de aulas expositivas, dadas por um membro da equipe (que utilizava recursos como vídeo, gravador, álbum seriado e dinâmica de grupo). Alguns adolescentes já haviam recebido informações sobre alguns temas desenvolvidos (através da mídia, escola, família ou amigos)

Os adolescentes, antes do início do programa, responderam a um questionário avaliativo (questionário de auto-aplicação, com questões do tipo certo ou errado, questões de múltipla escolha e questões dissertativas), com 16 questões relacionadas aos temas desenvolvidos subseqüentemente. Ao final do processo, os adolescentes responderam novamente o questionário avaliativo. $O$ confronto entre respostas pré e pós-programa serviu como aferição do conhecimento adquirido durante o desenvolvimento do mesmo.

A avaliação do programa sobre o nível de conhecimento dos adolescentes foi feita pelas diferenças entre as porcentagens de respostas corretas, dadas a cada pergunta nos questionários pré e pós-programa. Para essas comparações aplicou-se o teste de McNemar (Siegel, 1988) com o objetivo de se verificar as significâncias das referidas diferenças. Fixou-se em 0,05 o nível de significância.

\section{Resultados}

Os adolescentes estudados foram $37(62,7 \%)$ do sexo feminino e $22(37,3 \%)$ do sexo masculino com idade entre 12 e 19 anos, sendo a média e mediana de 13,5 para os adolescentes e 14 para as adolescentes. A maioria cursava o ensi- no fundamental II, sendo a $6^{\mathrm{a}}$ série a mais freqüentada.

Quando o assunto enfocado foram as fontes de informação sobre os componentes dos aparelhos reprodutores feminino e masculino, antes da realização do programa, o teste do Qui-quadrado mostrou diferença significante entre os sexos, predominando as informações de amigos para os adolescentes e livros para as adolescentes. A família informa mais as meninas do que os meninos.

\section{Avaliação do ganho de conhecimento}

Para avaliar o efeito do programa de orientação utilizou-se, como referencial, a porcentagem de respostas certas observadas nos períodos anterior e posterior à realização do programa.

Quando se procedeu à comparação de conhecimentos relacionados aos componentes do aparelho reprodutor feminino, o teste de McNemar mostrou que as porcentagens de respostas certas no período após o programa foram significantemente maiores que as observadas antes. Para o componente óvulo (Pré $=$ $45,9 \%$ e Pós $=89,2 \%$ ), para as adolescentes do sexo feminino e para os de sexo masculino (Pré $=59,1 \%$ e Pós $=90,9 \%)$ merecem ser notificados (Tabela 1).

Também para o aparelho reprodutor masculino houve aumento significante de conhecimento por parte dos adolescentes para todos os componentes, com exceção do pênis que teve $100,0 \%$ de acerto antes do programa (Tabela 2).

Quanto aos métodos contraceptivos citados, houve ganho de conhecimento por ambos os sexos, após o programa (Tabela 3). O método mais citado pelos adolescentes foi o preservativo $(95,4 \%)$, vindo a seguir a pílula $(81,8 \%)$. Para as adolescentes, os mais citados também foram o preservativo $(97,3 \%)$ e a pílula $(89,2 \%)$.

Em relação ao uso de preservativo, $45,9 \%$ das adolescentes, antes do programa, responderam que prevenia as doenças sexualmente transmissíveis e evitava a gravidez. Após o programa, 94,6\% responderam que ambas são evitadas. Para os adolescentes, antes do programa, $45,5 \%$ responderam que o uso do preservativo evitava as doenças sexualmente transmissíveis e, após o mesmo a porcentagem subiu para $100,0 \%$.

O número de drogas conhecidas foi maior no período após o programa, tanto para o sexo 
Tabela 1

Adolescentes do sexo feminino e masculino, segundo as porcentagens de respostas corretas, acerca dos componentes do aparelho reprodutor feminino, observadas nos períodos pré e pós-programa de orientação. Resultado do teste McNemar utilizado para comparação Pré versus Pós

\begin{tabular}{lccccrc}
\hline & \multicolumn{3}{c}{ Feminino } & \multicolumn{3}{c}{ Masculino } \\
Órgão & \multicolumn{3}{c}{ Respostas corretas } & \multicolumn{3}{c}{ Respostas corretas } \\
& Pré & Pós & Análise & Pré & Pós & Análise \\
\hline Trompas & 54,1 & 86,5 & $\mathrm{p}=0,0010^{*}$ & 54,5 & 86,4 & $0,0461^{*}$ \\
Útero & 72,9 & 97,3 & $\mathrm{p}=0,0020^{*}$ & 63,6 & 100,0 & $0,0039^{*}$ \\
Ovários & 64,9 & 89,2 & $\mathrm{p}=0,0020^{*}$ & 68,2 & 95,5 & $0,0352^{*}$ \\
Mamas & 94,6 & 100,0 & $\mathrm{p}=0,2500$ & 90,9 & 100,0 & 0,2500 \\
Óvulo & 45,9 & 89,2 & $\mathrm{p}=0,0000^{*}$ & 59,1 & 90,9 & - \\
Vagina & 100,0 & 100,0 & - & 100,0 & 100,0 & - \\
\hline
\end{tabular}

* Significante

Tabela 2

Adolescentes do sexo feminino e do masculino, segundo as porcentagens de respostas corretas, acerca dos componentes do aparelho reprodutor masculino, observadas nos períodos pré e pós-programa de orientação. Resultado do teste McNemar utilizado para comparação Pré versus Pós

\begin{tabular}{lcccccc}
\hline \multirow{2}{*}{ Órgãos } & \multicolumn{3}{c}{ Feminino } & \multicolumn{3}{c}{ Masculino } \\
& Pré & Respostas corretas & Respostas corretas \\
& & Pós & Análise & Pré & Pós & Análise \\
\hline Vesícula seminal & 29,7 & 86,5 & $\mathrm{p}=0,0000^{*}$ & 22,7 & 86,4 & $0,0013^{\star}$ \\
Espermatozóide & 73,0 & 92,0 & $\mathrm{p}=0,0195$ & 90,9 & 95,5 & 0,5000 \\
Glande & 27,0 & 83,8 & $\mathrm{p}=0,0000^{\star}$ & 18,2 & 68,2 & $0,0005^{\star}$ \\
Testículos & 59,5 & 97,3 & $\mathrm{p}=0,0000^{*}$ & 63,6 & 95,5 & $0,0018^{*}$ \\
Pênis & 97,3 & 100,0 & - & 100,0 & 100,0 & - \\
\hline
\end{tabular}

* Significante

\section{Tabela 3}

Adolescentes dos sexos feminino e masculino, atendidos no Centro de Saúde Escola da Universidade de Santo Amaro, segundo as porcentagens de respostas dadas em relação aos métodos contraceptivos, citados nas fases pré e pós-programa de orientação.

\begin{tabular}{|c|c|c|c|c|c|c|}
\hline \multirow[b]{2}{*}{ Método } & \multicolumn{3}{|c|}{ Feminino } & \multicolumn{3}{|c|}{ Masculino } \\
\hline & Antes & Após & Análise & Antes & Após & Análise \\
\hline Preservativo & 86,5 & 97,3 & 0,0625 & 81,8 & 95,4 & 0,1875 \\
\hline Pílula & 62,2 & 89,2 & $0,0010^{*}$ & 54,5 & 81,8 & 0,0547 \\
\hline DIU & 5,4 & 54,0 & $0,0000^{*}$ & 9,1 & 36,4 & 0,0547 \\
\hline Coito interrompido & 2,7 & 10,8 & 0,1250 & 4,5 & 18,2 & 0,1875 \\
\hline Diafragma & 2,7 & 29,7 & $0,0010^{*}$ & 4,5 & 27,3 & 0,0625 \\
\hline Vasectomia & 2,7 & 16,2 & $0,0312^{*}$ & 4,5 & 13,6 & 0,3125 \\
\hline Laqueadura & 8,1 & 16,2 & 0,1250 & 0,0 & 4,5 & - \\
\hline Tabelinha & 13,5 & 21,6 & 0,1250 & 0,0 & 4,5 & - \\
\hline Muco & 2,7 & 2,7 & 0,0 & 0,0 & - & - \\
\hline Espermaticida & 2,7 & 13,5 & 0,0625 & 9,1 & 13,6 & 0,500 \\
\hline
\end{tabular}

*Significante 
feminino quanto para o masculino (Tabela 4). As drogas mais citadas pelos adolescentes foram maconha $(90,9 \%)$, crack $(90,9 \%)$ e cocaína $(77,3 \%)$. Para as adolescentes os resultados foram a maconha $(97,3 \%)$, cocaína $(83,8 \%)$ e $\operatorname{crack}(64,9 \%)$.

O teste de McNemar mostrou também aumento significante na porcentagem de respostas "todas as alternativas" ao focalizar as vias de transmissão da Aids. Observou-se entre as mulheres um aumento de $7,3 \%$ para $35,5 \%$ e para os homens de $6,8 \%$ para $45,7 \%$.

\section{Discussão}

Os dados estatísticos confirmaram a baixa situação econômica dos pacientes que procuraram atendimento no Centro de Saúde Escola da Universidade de Santo Amaro.

Dados sobre a Pesquisa sobre Saúde Reprodutiva e Sexualidade do Jovem (PSRSJ, 1989. 1990) e de Sousa (1988) indicaram que os amigos têm papel importante na transmissão das primeiras noções sobre o sexo, tanto para os adolescentes como para as adolescentes, e estão de acordo com o que foi encontrado no presente estudo para os adolescentes do sexo masculino. Já para as adolescentes, os resultados estão de acordo com o que encontrou Pilon (1986), sendo os pais os preferidos pelas jovens como fonte de informação.
A sexualidade dos adolescentes, nas últimas décadas, revelou-se preocupante, devido a um incremento nacional bem fundamentado de gravidezes, doenças sexualmente transmissíveis e de outras complicações relacionadas ao exercício ativo da sexualidade.

Neste estudo pudemos observar que os adolescentes de ambos os sexos conheciam o preservativo e a pílula, antes das orientações fornecidas pelo programa. Este fato pode ter ocorrido, devido aos jovens terem recebido informações da escola, amigos e da própria família. Essa constatação (do conhecimento do preservativo e da pílula pelos jovens) foi feita quando a Pesquisa Sobre Saúde Reprodutiva e Sexualidade do Jovem (PSRSJ, 1989-1990) mostrou que a totalidade dos adolescentes conhecia, ou tinha ouvido falar sobre os dois métodos contraceptivos. Cabe ressaltar que os outros métodos contraceptivos foram pouco citados antes do programa, sendo conseqüentemente pouco conhecidos, fato que indica o pouco conhecimento dos mesmos pelos jovens. A ignorância da fisiologia da reprodução e das conseqüências das relações sexuais por parte de muitas adolescentes e o fato de que a contracepção continua sendo uma fonte de dificuldade para elas parecem constituir alguns dos principais obstáculos que se opõem a seu uso neste grupo etário.

Fora do meio familiar existem poucos serviços disponíveis para orientar e informar os

Tabela 4

Porcentagem de drogas conhecidas, citadas por adolescentes dos sexos masculino e feminino, antes e depois do programa de orientação.

\begin{tabular}{lcccrrr}
\hline \multirow{2}{*}{ Droga } & \multicolumn{3}{c}{$\begin{array}{c}\text { Feminino } \\
\text { \% drogas conhecidas }\end{array}$} & \multicolumn{3}{c}{$\begin{array}{c}\text { Masculino } \\
\text { \% drogas conhecidas }\end{array}$} \\
& Antes & Após & $\mathrm{p}$ & Antes & Após & $\mathrm{p}$ \\
\hline Maconha & 59,5 & 97,3 & $0,0000^{*}$ & 81,8 & 90,9 & 0,3438 \\
Cocaína & 73,0 & 83,8 & 0,0625 & 59,0 & 77,3 & 0,1938 \\
Crack & 64,9 & 64,9 & - & 77,3 & 90,9 & 0,2266 \\
LSD & 5,4 & 10,8 & 0,2500 & 0,0 & 9,1 & 0,2500 \\
Heroína & 10,8 & 27,0 & $0,0156^{*}$ & 22,7 & 36,4 & 0,2744 \\
Cola & 24,3 & 35,5 & 0,0625 & 18,2 & 22,7 & 0,5000 \\
Remédio & 2,7 & 13,5 & 0,0625 & 0,0 & 0,0 & - \\
Cigarro & 2,7 & 37,8 & $0,0001^{*}$ & 18,2 & 36,4 & 0,1938 \\
Álcool & 2,7 & 37,8 & $0,0001^{*}$ & 13,6 & 31,8 & 0,1445 \\
Esmalte & 2,7 & 16,2 & $0,0312^{*}$ & 0,0 & 0,0 & - \\
Xarope & 0,0 & 2,7 & - & 0,0 & 4,5 & - \\
Êxtase & 0,0 & 2,7 & - & 4,5 & 9,1 & - \\
\hline
\end{tabular}

*Significante 
jovens quanto à sexualidade e anticoncepção. Muitas vezes os serviços de planejamento familiar geralmente se mostram inúteis para os adolescentes, pois concentram-se na cobertura de casais idosos e com muitos filhos, não se estruturando de forma adequada às características peculiares desse grupo etário, que requer uma atenção particular e especializada.

Em nosso estudo verificamos que após o programa, a grande maioria passou a considerar importante o uso do preservativo, e respondeu que o seu uso prevenia a gravidez e as doenças sexualmente transmissíveis.

Quanto às drogas mais conhecidas pelos adolescentes, ficou evidente em nosso estudo, que foram citadas as proibidas e sujeitas a um controle policial (crack, cocaína e maconha). Benguigui et al. (1977) relataram o uso excessivo de álcool pelos adolescentes e também o aumento de tabaco entre mulheres jovens. No atual estudo essas drogas foram pouco citadas, principalmente pelas adolescentes. Esse fato pode ter ocorrido devido ao desconhecimento dos efeitos maléficos das mesmas por grande parte da população, já que são menos estigmatizadas e sujeitas a preconceitos. Deve ser enfatizado que, muitas vezes o jovem inicia sua carreira de viciados por elas, como Scivoletto et al. (1996) descreveram. Deveria se pensar em produzir mais campanhas educativas e utilizar a mídia em horários propícios para o público jovem, como forma de conscientizar e informar essa faixa etária. As escolas também deveriam ser mais atuantes e informar mais os jovens, pois de qualquer maneira eles estão hoje submetidos à cultura das drogas.

Deve ser ressaltado que antes da realização do programa de orientação a maioria dos adolescentes vinculava a Aids às vias de transmissão pelas relações sexuais e pela corrente san- güínea, referindo-se poucas vezes às demais vias de transmissão. Chicrala et al. (1977) encontraram em seus estudos dados semelhantes. Em nosso estudo, com a aplicação do programa, os jovens aprimoraram seus conhecimentos e passaram a citar outras vias de transmissão (o uso de drogas injetáveis e amamentação). Houve ainda ganho de conhecimento quanto a considerar a Aids uma doença sexualmente transmissível. Estamos de acordo com Ribeiro (1988) quando afirma que a família tem um papel fundamental na educação dos filhos para a prevenção de doenças sexualmente transmissíveis/Aids. Percebemos que nas classes sociais mais baixas os pais também não têm informações sobre essas doenças e não conversam com os filhos sobre sexualidade de uma maneira geral, o que indica a necessidade de criar equipes multidisciplinares de orientação que venham preencher a lacuna.

\section{Conclusões}

Os adolescentes adquiriram conhecimentos sobre os temas desenvolvidos, referentes a questões específicas dessa fase de desenvolvimento, por intermédio de uma equipe multiprofissional.

a) Quando comparados, os conhecimentos prévios sobre os temas desenvolvidos no Programa de Orientação e os conhecimentos ao final do programa, foi possível concluir que houve ganho significante para a grande maioria das questões abordadas.

b) Tanto os adolescentes do sexo masculino como os do sexo feminino foram beneficiados com as orientações e não diferiram significantemente em relação aos conhecimentos adquiridos com o programa. 


\section{Referências bibliográficas}

Aberastury A \& Knobel M 1992. Adolescência normal. Artes Médicas, Porto Alegre.

Benguigui Y, Land S, Paganini JM \& Yunes J 1997. Ações de saúde materno-infantil em nível local: segundo as metas da Cúpula Mundial em Favor da Infância. Comitê Coordenador Interagencial para as Américas/ Opas, Washington.

Braden PS 2000. Enfermagem materno-infantil. Reichman \& Affonso Editores, Rio de Janeiro.

Chicrala MA et al. 1977. Conhecimento, atitudes e práticas relacionadas à DST/Aids. Avaliação de adolescentes atendidos em Unidade de Atenção Primária. Jornal Brasileiro de Doenças Sexualmente Transmissíveis 9(3):10-15.

Coli AS 1994. Conceito de adolescência. In E Marcondes. Pediatria básica. Savier, São Paulo.

Comissão de Saúde do Adolescente 1994. Adolescência e saúde. Ed. São Paulo-Paris Editorial - Secretaria do Estado da Saúde, São Paulo.

Donas S 1992. Marco epidemiológico-conceptual de saúde integral do adolescente. Organização Mundial de Saúde, Brasma, Brasil.
Messina M 1993. Programa de saúde do adolescente em Blumenau. Arquivos Catarinenses Médicos 22(1/2): 17-20.

Osório LC 1989. Adolescente hoje. Artes Médicas, Porto Alegre.

Pilon AF 1986. O jovem e seu projeto de vida. Revista Saúde Pública (20):244-250.

Ribeiro MA 1998. Comunicação familiar e prevenção de DSTs/AIDS entre adolescentes. Jornal Brasileiro de Doenças Sexualmente Transmissíveis 10(1):5-9.

Scivoletto S, Jr. SGA \& Andrade AG 1996. A progressão do consumo de drogas entre adolescentes que procuraram tratamento. Jornal Brasileiro de Psiquiatria 45 (4):201-207.

Siegel S, Castellan Jr. \& NJ 1988. Non-parametrics statistics. (2a ed.). Mc Graw Hill. Nova York.

Sociedade Civil Bem-Estar Familiar no Brasil-Bemfam 1990. Pesquisa sobre saúde reprodutiva e sexualidade do jovem. Rio de Janeiro-Curitiba-Recife.

Sousa RP 1988. Coletânea sobre saúde reprodutiva do adolescente brasileiro. Opas/OMS, Brasília-DF.

Artigo apresentado em 17/2/2003

Aprovado em 31/3/2003

Versão final apresentada em 30/5/2003 